\title{
The impact of atomistic substitution on thin-film structure and charge transport in a germanyl-ethynyl functionalized pentacene
}

Jeni C. Sorli, Qianxiang Ai, Devin B. Granger, Kaichen Gu, Sean Parkin, Karol Jarolimek, Nicholas Telesz, John E. Anthony, Chad Risko, Yueh-Lin Loo

\section{Materials}

TIPGe-Pn, for which synthetic details have been previously reported, ${ }^{1,2}$ was obtained from Prof. John Anthony's group at the University of Kentucky. All solvents were purchased from Fischer-Scientific and used as received. Material requests should be directed to Prof. John Anthony.

\section{Crystal Growth}

Purified TIPGe-Pn exhibits the herringbone packing motif when grown from solutions of hexanes, acetone, ethyl acetate, toluene. In order to grow large crystals (i.e., of sufficient size and stability to pry off the growth substrate and perform X-ray analysis) of the brickwork packing motif, we allowed a solution of TIPGe-Pn in toluene to fully evaporate in the dark at ambient conditions. The resulting solid was re-dissolved in a 1:1:1 mixture of butyl acetate, toluene and heptane at room temperature, and the solution allowed to again evaporate slowly in a crystallizing dish in the dark. This process was repeated until the crystals on the surface were large enough to be scraped off and submitted for $\mathrm{X}$-ray analysis. Needle-like crystals of the slip-stack polymorph also formed during this process, but only crystals in the brickwork polymorph formed in substantial quantities.

\section{Substrate Preparation}

Si wafers $(\mathrm{Si}(100))$ with thermally grown $\mathrm{SiO}_{2}(300 \mathrm{~nm})$ were purchased from Process Specialties Inc., and used as the gate and gate dielectric, respectively, for thin-film transistors and for GIXD studies. The substrates were sonicated in dionized water, acetone, and isopropyl alcohol and then dried with nitrogen prior to transistor fabrication. For GIXD studies, films were prepared on cleaned, untreated $\mathrm{Si} / \mathrm{SiO}_{2}$ substrates. For transistors, bottom contacts were prepared by depositing $2 \mathrm{~nm}$ of titanium, followed by $30 \mathrm{~nm}$ of gold through 
pre-defined stencil masks. These patterned substrates were immediately placed in a $7.5 \mathrm{mM}$ PFBT/ethanol solution for 20 minutes upon removal from vacuum, then sonicated in ethanol and dried with nitrogen before deposition of the organic semiconductors. For UV-visible measurements, glass slides were cleaned with the same procedure described above for silicon wafers.

\section{Thin-Film Deposition}

TIPGe-Pn thin films were made by spin-coating a $10 \mathrm{mg} / \mathrm{mL}$ chloroform solution at $1500 \mathrm{rpm}$ for 60 seconds under ambient conditions. TIPGe-Pn ribbons were made by aligned drop-casting ${ }^{3}$ a $5 \mathrm{mg} / \mathrm{mL}$ heptane solution onto silicon wafers or PFBT-treated gold patterns for the production of bottomcontact transistors. Glass slides treated with Cytop were used as the upper pinning surface during aligned drop-casting, in which the bottom edge of the glass slide was in contact with the wafer and the spacer height at the leading edge was $150 \mu \mathrm{m}$ for solution injection, as described by Nakayama et al. ${ }^{3}$ The substrates and solution were brought to $65^{\circ} \mathrm{C}$ on a hot plate, and the solution was injected between the substrate and the pinning surface. Evaporation of the solvent led to preferentially aligned ribbons of TIPGe-Pn.

\section{Post-Deposition Processing}

Solvent-vapor annealing was performed by placing TIPGe-Pn films in a covered Petri dish at room temperature. A solvent reservoir with $10 \mathrm{~mL}$ of toluene was placed beside the sample in the Petri dish. ${ }^{4-7}$ As toluene vapor plasticized TIPGe-Pn films, phase transformation took place within 15 minutes in this setup.

\section{Device Fabrication and Characterization}

Bottom-contact (PFBT treated $\mathrm{Au}$ ), bottom-gate (Si) thin-film transistors with a 300-nm thick $\mathrm{SiO}_{2}$ dielectric layer were fabricated with TIPGe-Pn thin films. The channel length and width for TIPGe-Pn devices were 50 and $200 \mu \mathrm{m}$, respectively. While the ribbons produced from aligned drop-casting spanned the channels, they did not completely cover the channel width. As such, the channel of each tested device was imaged using an optical microscope and the effective channel width estimated in ImageJ. All transistors were tested in air 
using an Agilent 4155C semiconductor parameter analyzer. The hole mobilities were estimated in the saturation regime at $V_{\mathrm{SD}}=-80 \mathrm{~V}$. Threshold voltages were calculated by extrapolating the zero point of the square root of current versus the gate voltage in the saturation regime. On/off current ratios were calculated by taking the ratio of $I_{S D}$ at $-80 \mathrm{~V}$ (on) and at the turn-on voltage (off) from the transfer characteristics.

\section{Structural Characterization}

GIXD experiments were conducted at the Cornell High Energy Synchrotron Source on the G1 station $(9.95 \pm 0.05 \mathrm{keV})$. The X-ray beam was aligned between the critical angles of the film and substrate, at $0.17^{\circ}$ relative to the substrate surface. The scattered $x$-rays was collected on a two-dimensional CCD detector, and all GIXD images have been background subtracted. Background subtracted GIXD patterns of aligned drop-cast films were fit using DPC toolkit ${ }^{8}$ and the obtained crystal structure subsequently minimized via DFT to obtain the slip-distances (Table S2 and S3). UV-visible spectra were collected on samples prepared on glass slides and measured using an Agilent Technologies Cary 5000 spectrophotometer. Spectra have been background subtracted. Film thickness measurements were performed using ellipsometry for spun-cast films on a Woollam M-2000 Ellipsometer and using atomic force microscopy (AFM) for drop-cast films on a Bruker Dimension ICON3 Atomic Force Microscope.

\section{Computational Details}

DFT calculations were carried out with Vienna Ab-initio Simulation Package (VASP), ${ }^{9-12}$ making use of the Perdew, Burke, and Ernzerhof (PBE) exchangecorrelation functional. ${ }^{13}$ The electron-ion interactions were described with the projector augment wave (PAW) method. ${ }^{14}$ The kinetic energy cutoff for the plane-wave basis set was set to $520 \mathrm{eV}$, and a Gaussian smearing with a width of $50 \mathrm{meV}$ was employed. The D3 correction was employed with BJ-damping to describe the dispersion forces. ${ }^{15,16}$ The convergence criterion of the total energy was set to $10^{-5} \mathrm{eV}$ in the self-consistent field loop, and that of forces during relaxation was set to $0.01 \mathrm{eV} / \AA$. 
The cohesive energy of a periodic structure $S, E_{S}$, is defined by $E_{S}=-\frac{E_{S}^{P B E}-N \cdot E_{m o l}^{P B E}}{N}$, where $E_{S}^{P B E}$ is the total energy per unit cell from DFT calculations, $E_{m o l}^{P B E}$ is the total energy of a single molecule, $\mathrm{N}$ is the number of molecules per unit cell. 
Figures
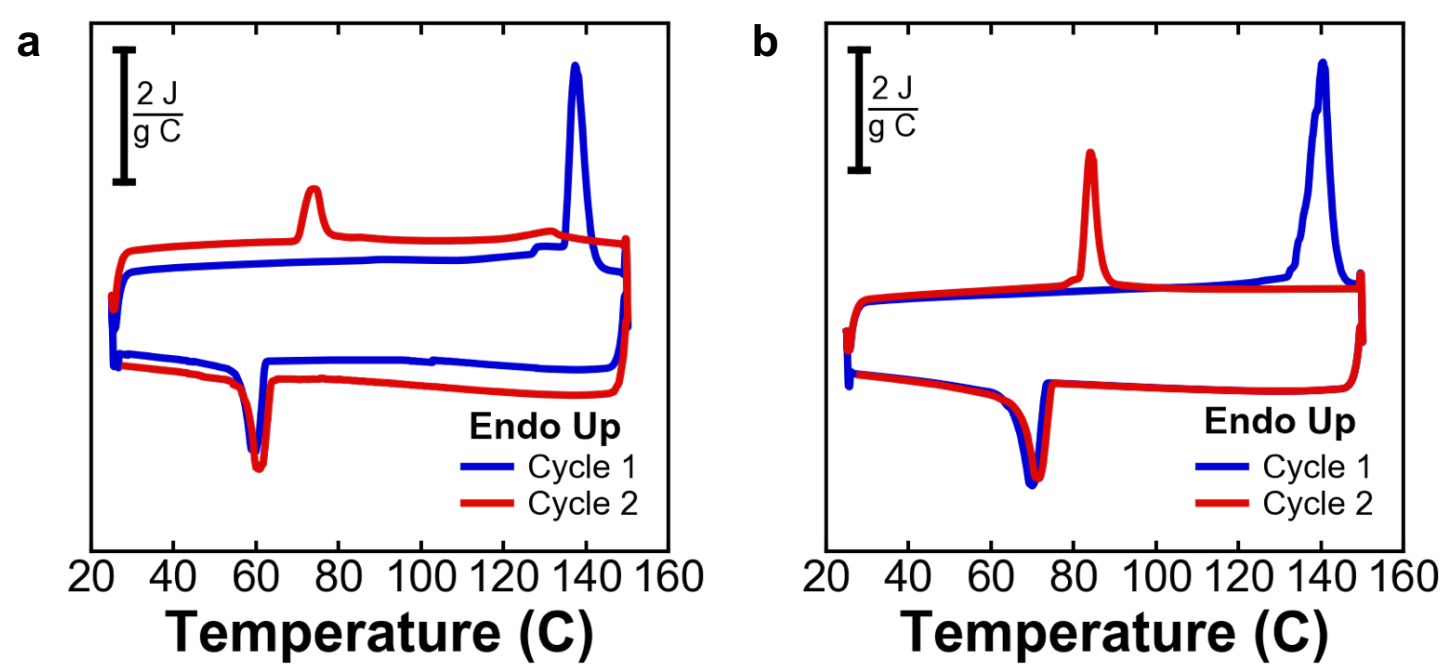

Figure S1. Differential scanning calorimetry (DSC) thermograms of TIPGe-Pn single crystals having the (a) brickwork and (b) herringbone packing motifs. 


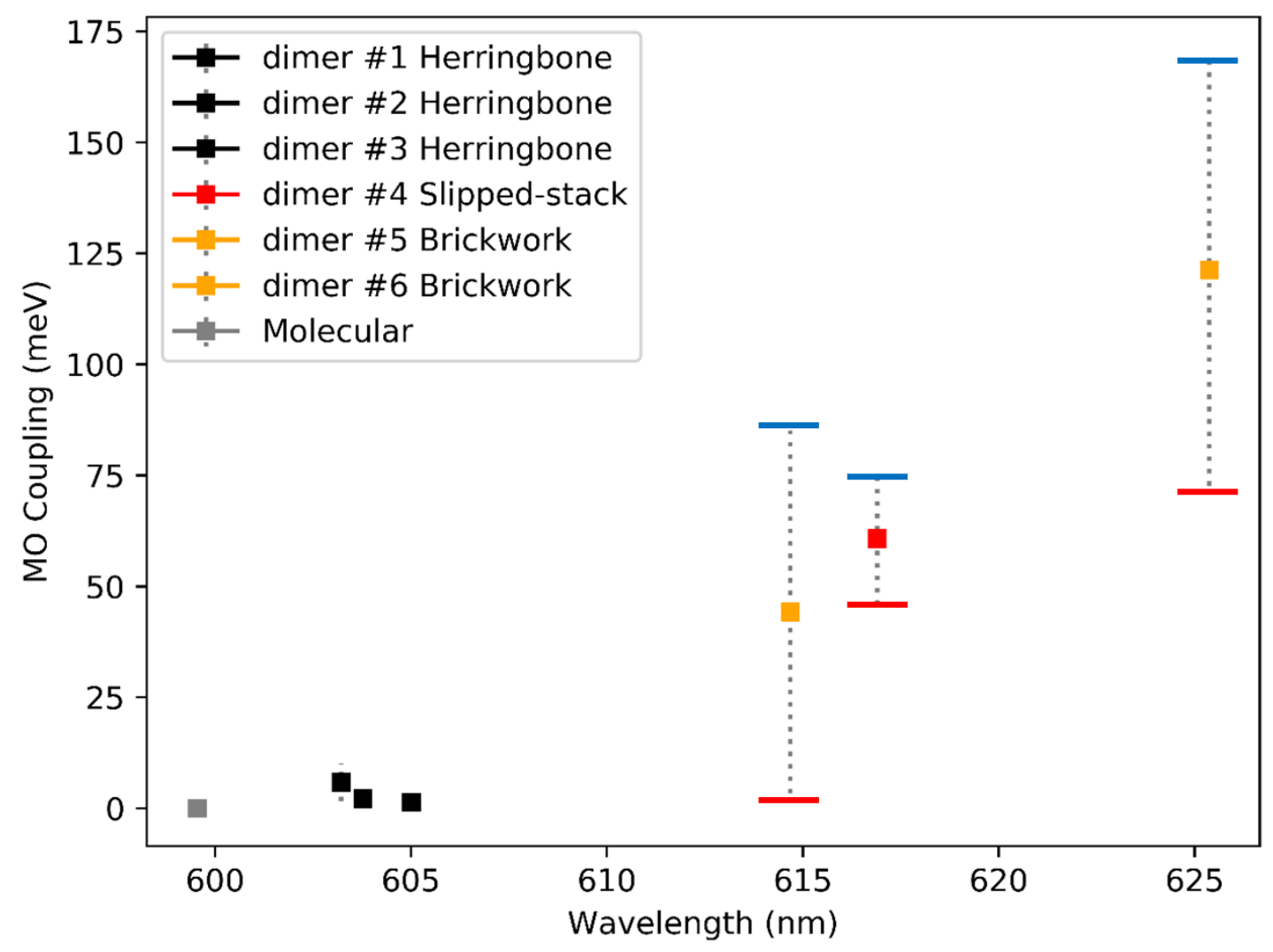

Figure S2. Positive correlation between the wavelength of the first bright state ( $\lambda$ max) and intermolecular coupling, both calculated from dimer models extracted from different TIPGe-Pn polymorphic structures, expect for the gray point which represents unimolecular $\lambda_{\max }$. If multiple dimer models are available for a certain polymorph, only the one with largest $\lambda_{\max }$ is included. All calculations here are done at wb97xd/def2svp level with excited states calculated with TDDFT routines. 

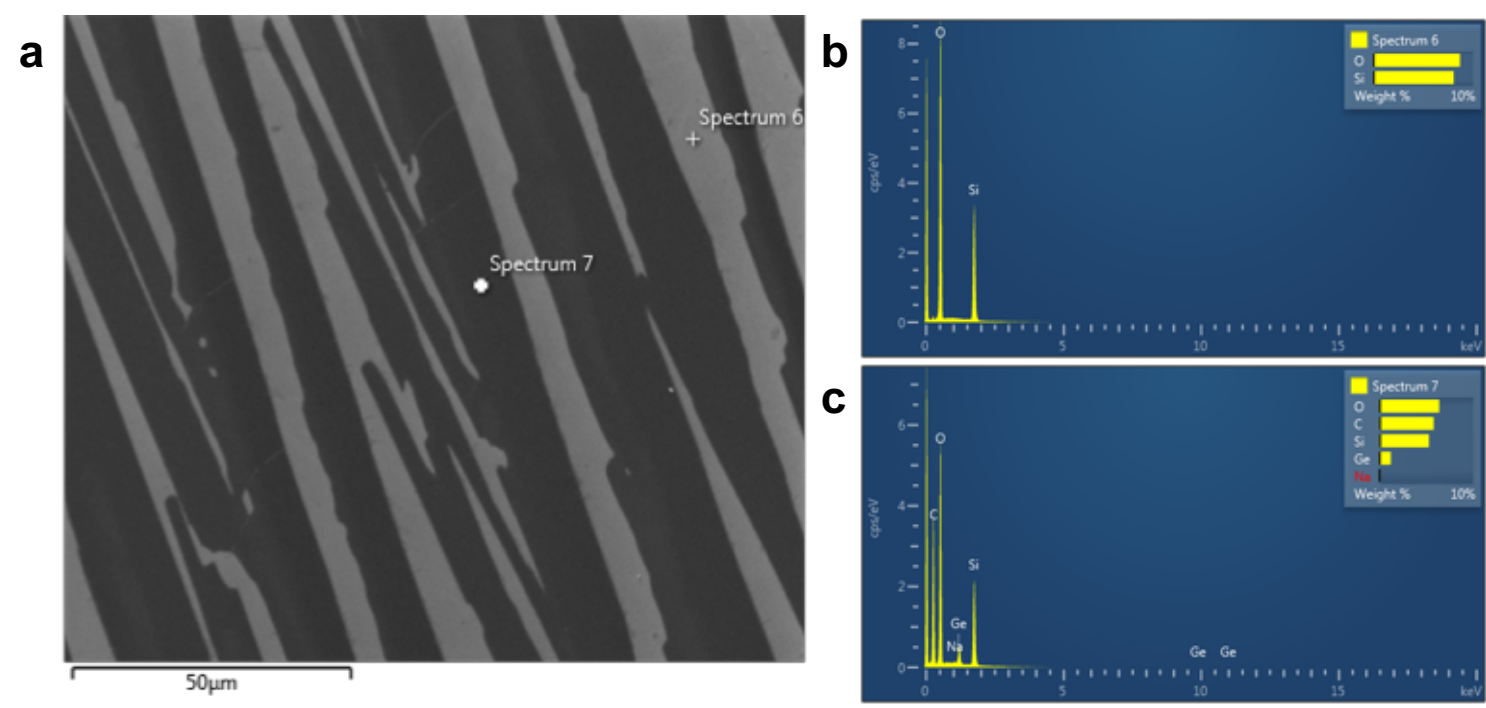

Figure S3. (a) SEM image of TIPGe-Pn aligned drop-cast films and respective EDX spectra acquired on the (b) light and (c) dark regions of the image. 

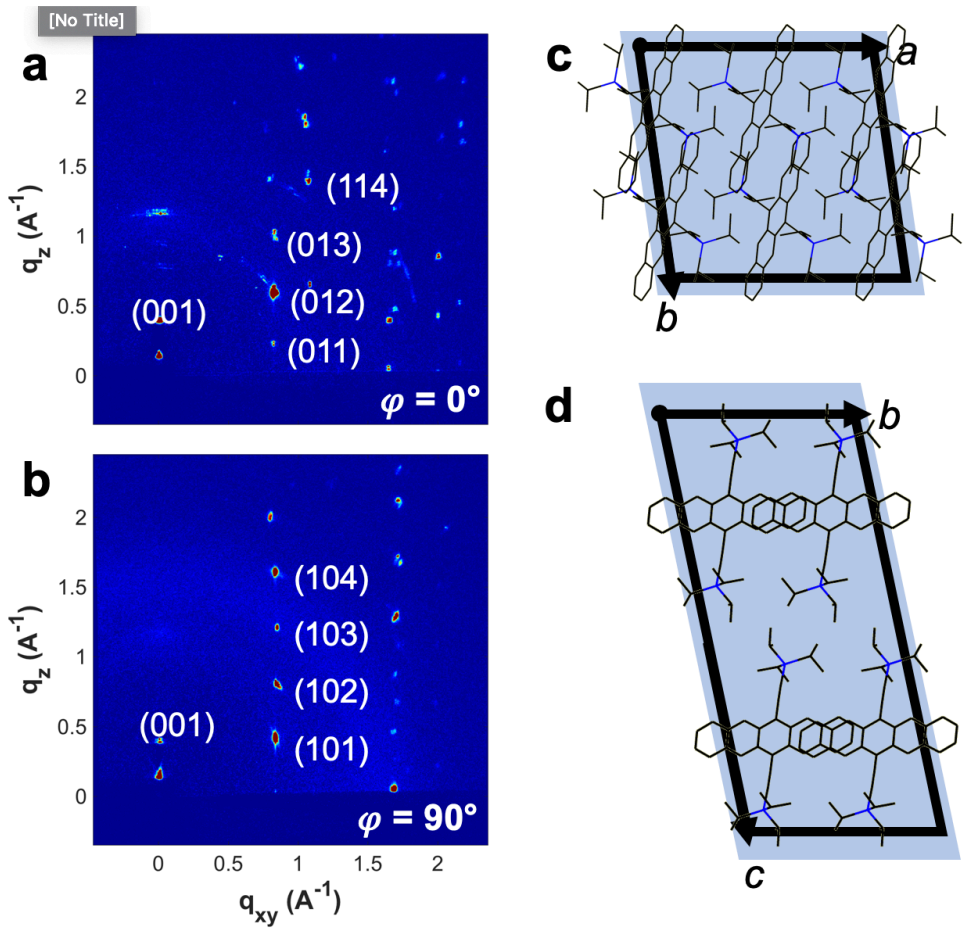

Figure S4. 2D GIXD patterns of TIPGe-Pn ribbons with incident x-rays (a) normal and (b) parallel to their long axis corresponding to the perspectives in (c) and (d). (c) Unit cell of TIPGe-Pn aligned drop-cast crystals viewed along the c-axis, corresponding to a top-down view of the ribbons, in which the $\pi$ stacking direction is oriented with the long axis of the ribbon. (d) The same unit cell viewed along the a-axis (viewed along the long axis of the ribbons). Unit cell dimensions for aligned drop-cast films were obtained using DPC toolkit. ${ }^{8}$ 

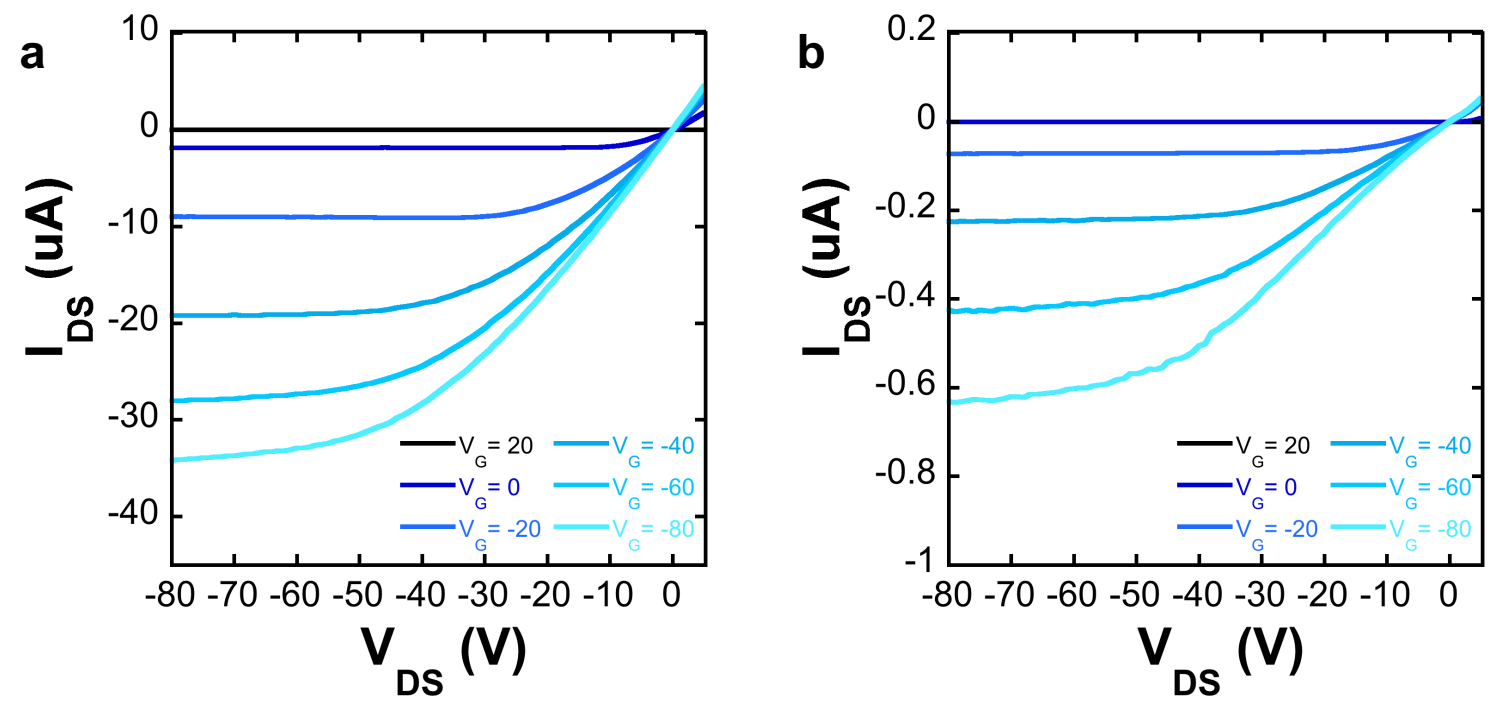

Figure S5. Representative output curves for thin-film transistors with active layers of TIPGe-Pn (a) aligned drop-cast ribbons and (b) TA spun-cast films. 


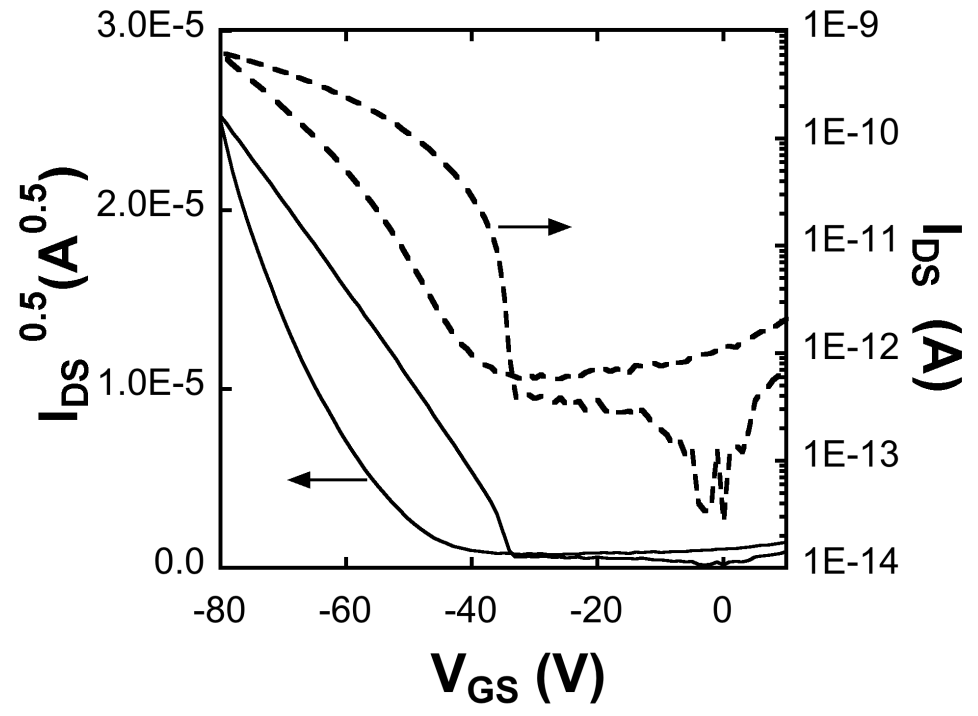

Figure S6. Representative transfer curve for spun-cast, TA TIPGe-Pn thin film OTFTs, with $V_{D S}=-80 \mathrm{~V}$. 
Table S1. Bulk crystal structure lattice parameters for TIPGe-Pn obtained by single crystal X-ray diffraction.

\begin{tabular}{|l|l|l|l|l|l|l|l|l|}
\hline $\begin{array}{l}\text { Packing } \\
\text { Motif }\end{array}$ & CCDC No. & $\mathbf{a}(\boldsymbol{\AA})$ & $\mathbf{b}(\boldsymbol{\AA})$ & $\mathbf{c}(\boldsymbol{\AA})$ & $\left.\boldsymbol{\alpha} \mathbf{(}^{\circ}\right)$ & $\left.\boldsymbol{\beta} \mathbf{(}^{\circ}\right)$ & $\gamma\left(\mathbf{(}^{\circ}\right)$ & $\begin{array}{l}\text { Space } \\
\text { group }\end{array}$ \\
\hline Herringbone & 1889788 & 14.4240 & 14.7388 & 18.0870 & 90 & 90 & 90 & Pbca \\
\hline Slip-stack & 1889787 & 8.5753 & 10.8951 & 10.9628 & 67.029 & 87.521 & 87.155 & $\mathrm{P} \overline{1}$ \\
\hline Brickwork & 1889786 & 7.7283 & 7.7628 & 16.8805 & 78.077 & 89.228 & 81.788 & $\mathrm{P} \overline{1}$ \\
\hline
\end{tabular}


Table S2. Elemental compositions from EDX scans of the light and dark regions of aligned drop-cast TIPGe-Pn ribbons on $\mathrm{SiO}_{2}$.

\begin{tabular}{ccc}
\hline Spectra & Element & Atomic \% \\
\hline $\begin{array}{c}\text { Bare } \mathrm{SiO}_{2} \\
(\text { Spectrum 6) }\end{array}$ & $\mathrm{Si}$ & 34.60 \\
\cline { 2 - 3 } & $\mathrm{O}$ & 65.40 \\
\hline TIPGe-Pn Ribbon on & $\mathrm{Si}$ & 17.29 \\
SiO $_{2}$ & $\mathrm{O}$ & 36.76 \\
$($ Spectrum 7) & $\mathrm{C}$ & 44.37 \\
\cline { 2 - 3 } & $\mathrm{Ge}$ & 1.55 \\
& $\mathrm{Na}$ & 0.04 \\
\hline
\end{tabular}


Table S3. Solved unit cell parameters for TIPGe-Pn ribbons.

\begin{tabular}{|l|l|l|l|l|l|l|l|}
\hline Processing & $\mathbf{a}(\boldsymbol{(})$ & $\mathbf{b}(\boldsymbol{\AA})$ & $\mathbf{c}(\AA)$ & $\alpha\left(^{\circ}\right)$ & $\beta\left(^{\circ}\right)$ & $\gamma\left(^{\circ}\right)$ & $\begin{array}{l}\text { Space } \\
\text { group }\end{array}$ \\
\hline Brickwork & 7.7283 & 7.7628 & 16.8805 & 78.077 & 89.228 & 81.788 & $\mathrm{P} \overline{1}$ \\
\hline As-cast & 7.69 & 7.79 & 16.10 & 78.4 & 88.3 & 80.1 & $\mathrm{P} \overline{1}$ \\
\hline TA & 8.09 & 7.81 & 16.43 & 77.7 & 88.6 & 82.3 & $\mathrm{P} \overline{1}$ \\
\hline SVA & 8.03 & 7.96 & 16.50 & 77.7 & 88.4 & 81.8 & $\mathrm{P} \overline{1}$ \\
\hline
\end{tabular}

Solved unit cell parameters were obtained using DPC toolkit. ${ }^{8}$ The brickwork structure is experimentally obtained from single crystals, and officially logged as CCDC 1889786. 
Table S4. Comparison of TIPGe-Pn slip distances along different axes between the unit cell exhibiting the brickwork packing motif and the solved crystal structure of aligned drop-cast films from DFT.

\begin{tabular}{|l|l|l|l|}
\hline Structure & $\begin{array}{l}\text { Long Axis Slip } \\
(\boldsymbol{\AA})\end{array}$ & $\begin{array}{l}\text { Short Axis Slip } \\
(\mathbf{\AA})\end{array}$ & $\begin{array}{l}\text { Vertical Slip } \\
(\mathbf{\AA})\end{array}$ \\
\hline TIPGe-Pn Drop-cast & 9.24 & 1.94 & 3.20 \\
& 6.91 & 1.39 & 3.34 \\
\hline TIPGe-Pn Brickwork & 9.43 & 1.83 & 3.26 \\
\cline { 2 - 4 } & 6.89 & 1.24 & 3.36 \\
\hline
\end{tabular}




\section{References}

(1) Anthony, J. E.; Eaton, D. L.; Parkin, S. R. A Road Map to Stable, Soluble, Easily Crystallized Pentacene Derivatives. Org. Lett. 2002, 4, 15-18.

(2) Zhang, H.; Yao, Y.; Payne, M. M.; Anthony, J. E.; Brill, J. W. Thermal Diffusivities of Functionalized Pentacene Semiconductors. Appl. Phys. Lett. 2014, 105, 073302.

(3) Nakayama, K.; Hirose, Y.; Soeda, J.; Yoshizumi, M.; Uemura, T.; Uno, M.; Li, W.; Kang, M. J.; Yamagishi, M.; Okada, Y.; et al. Patternable Solution-Crystallized Organic Transistors with High Charge Carrier Mobility. Adv. Mater. 2011, 23, 1626-1629.

(4) Hiszpanski, A. M.; Baur, R. M.; Kim, B.; Tremblay, N. J.; Nuckolls, C.; Woll, A. R.; Loo, Y.-L. Tuning Polymorphism and Orientation in Organic Semiconductor Thin Films via Post-Deposition Processing. J. Am. Chem. Soc. 2014, 136, 15749-15756.

(5) Hiszpanski, A. M.; Lee, S. S.; Wang, H.; Woll, A. R.; Nuckolls, C.; Loo, Y.-L. Post-Deposition Processing Methods To Induce Preferential Orientation in Contorted Hexabenzocoronene Thin Films. ACS Nano 2012, 7, 294-300.

(6) Hiszpanski, A. M.; Woll, A. R.; Kim, B.; Nuckolls, C.; Loo, Y.-L. Altering the Polymorphic Accessibility of Polycyclic Aromatic Hydrocarbons with Fluorination. Chem. Mater. 2017, 29, 4311-4316.

(7) Purdum, G. E.; Yao, N.; Woll, A. R.; Gessner, T.; Weitz, R. T.; Loo, Y.-L. Understanding Polymorph Transformations in Core-Chlorinated Naphthalene Diimides and Their Impact on Thin-Film Transistor Performance. Adv. Funct. Mater. 2016, 26, 2357-2364.

(8) Hailey, A. K.; Hiszpanski, A. M.; Smilgies, D. M.; Loo, Y.-L. The Diffraction Pattern Calculator (DPC) Toolkit: A User-Friendly Approach to Unit-Cell Lattice Parameter Identification of Two-Dimensional GrazingIncidence Wide-Angle X-Ray Scattering Data. J. Appl. Crystallogr. 2014, 47, 2090-2099. 
(9) Kresse, G.; Hafner, J. Ab Initio Molecular Dynamics for Liquid Metals. Phys. Rev. B 1993, 47, 558-561.

(10) Kresse, G.; Furthmüller, J. Efficiency of Ab-Initio Total Energy Calculations for Metals and Semiconductors Using a Plane-Wave Basis Set. Comput. Mater. Sci. 1996, 6, 15-50.

(11) Kresse, G.; Furthmüller, J. Efficient Iterative Schemes for Ab Initio TotalEnergy Calculations Using a Plane-Wave Basis Set. Phys. Rev. B Condens. Matter Mater. Phys. 1996, 54, 11169-11186.

(12) Kresse, G.; Joubert, D. G. Kresse and D. Joubert, Phys. Rev. B 59, 1758 (1999). Phys. Rev. B 1999, 59, 1758.

(13) Perdew, J. P.; Chevary, J. A.; Vosko, S. H.; Jackson, K. A.; Pederson, M. R.; Singh, D. J.; Fiolhais, C. Atoms, Molecules, Solids, and Surfaces: Applications of the Generalized Gradient Approximation for Exchange and Correlation. Phys. Rev. B 1992, 46, 6671-6687.

(14) Blöchl, P. E. Projector Augmented-Wave Method. Phys. Rev. B 1994, 50, 17953-17979.

(15) Grimme, S.; Antony, J.; Ehrlich, S.; Krieg, H. A Consistent and Accurate $A b$ Initio Parametrization of Density Functional Dispersion Correction (DFT-D) for the 94 Elements H-Pu. J. Chem. Phys. 2010, 132, 154104.

(16) Grimme, S.; Ehrlich, S.; Goerigk, L. Effect of the Damping Function in Dispersion Corrected Density Functional Theory. J. Comput. Chem. 2011, $32,1456-1465$. 